\title{
TIPOS DE RACIONALIDAD
}

\author{
Herbert Schnädelbach \\ Universidad Humboldt de Berlin \\ (Traducción de José Luis López y López de Lizaga)
}

Resumen: Este escrito traza el esbozo de una teoría de la racionalidad que intenta analizar el concepto de racionalidad del modo más comprehensivo y completo posible. No sólo se tendrán en cuenta los más importantes contextos de aplicación de este concepto en la actualidad, sino también aquellos otros de la tradición filosbfica, que en su corriente principal siempre se ha interpretado a si misma como filosofia de la razb́n. Por supuesto, esto sólo es posible empleando medios tipológicos. La explicación propuesta distingue entre racionalidad de fundamentación en el nivel del lenguaje objeto, racionalidad discursiva (argumentación) y racionalidad de reglas, y señala subtipos en cada uno de estos niveles. De este modo, nuestra explicación se orienta por la distinción kantiana entre razón, facultad de juzgar y entendimiento. Finalmente, el concepto de intencionalidad como condición elemental de la racionalidad establece la conexión entre la teoria de la racionlidad y la antropología filosófica.

(Advertencia preliminar: Como en lo que sigue sólo intento presentar los fundamentos de una teoría, renuncio a exponer posiciones alternativas y a citar pormenorizadamente las fuentes bibliográficas. La discusion de este texto no debería referirse a si he comprendido o apreciado correctamente a otros autores, a no ser que de ello surgiese algún argumento acerca del asunto mismo de que se trata).

\section{Introducción}

((0.1)) En las dos últimas décadas ha aparecido un gran número de monografías, publicaciones colectivas y artículos en cuyos títulos aparece la 
palabra "racionalidad", o que se refieren directamente a lo que esta palabra designa. Parece que, tras una época que ha durado casi un siglo y medio y en la que ha imperado entre los filósofos la crítica de la razón (desde la muerte de Hegel y bajo la influencia de la historia de los efectos de Schopenhauer y Nietzsche), ha llegado la hora de que éstos se ocupen de nuevo, de forma constructiva o reconstructiva, del tema fundamental "logos, ratio, intellectus, entendimiento, razón", tan denostado en la época anterior. Durante demasiado tiempo pareció intuitivamente claro contra qué se dirigian los ataques de la crítica de la razón, y a esta vaguedad ha de atribuirse, y no en último lugar, el hecho de que este programa aunase los esfuerzos de tantos y tan diversos espíritus. Por supuesto, este giro no ha de agradecerse a un espontáneo cambio de sensibilidades de los filósofos, que al menos en Alemania y Francia continuarían gustosamente con sus letanías nietzscheanas, sino que es el resultado de la necesidad urgente de reaccionar a algo que ha sucedido fuera de los estrechos muros de la filosoffa. Sobre todo en las ciencias sociales, el tema de la racionalidad ha llegado a ser un tema de actualidad: primero en la economía (desde J. St. Mill), después en la teoría sociológica de la acción (Max Weber y sus seguidores), en la metodología de las ciencias sociales (bajo el rótulo de «teoría de las explicaciones racionales"), en la etnología (véase la controversia de Winch); pero también, más tarde, en la teoría de la ciencia (de Popper a Feyerabend), que durante mucho tiempo fue considerada en los seminarios filosóficos tradicionales como una disciplina propiamente extrafilosófica. Cuando también los filósofos comenzaron a interesarse por los resultados obtenidos entretanto en estos ámbitos diversos por lo que respecta a las concepciones de la racionalidad, se les ofreció un cuadro bastante confuso. Por una parte se vieron ante un problema de armonización, pues el término "racionalidad" se atribuía a cosas bastante heterogéneas, cuyo denominador común no era reconocible; por otra parte no era posible reencontrar en los proyectos existentes de una teoría de la racionalidad simplemente los objetos de la filosofía tradicional de la razón, de modo que parecía que había que tratar con algo enteramente nuevo.

((0.2)) Parto del supuesto de que este tránsito de la filosofía de la razón a la teoría filosófica de la racionalidad no tiene vuelta atrás, pero creo, al mismo tiempo, que con él se pueden explicar los dos defectos principales de casi todas las teorías existentes de la racionalidad. Por un lado, la fijación de un contexto de introducción del concepto de racionalidad tomado de una determinada ciencia particular. Gran parte de la discusión sobre la racionali- 
dad en el ámbito anglosajón está completamente determinada por los problemas estructurales y de detalle de la racionalidad económica (teoría de la decisión y teoría de juegos). En otros casos domina en la bibliografía dedicada a esta cuestión la "racionalidad de fines" de Max Weber, convertida en fetiche, que no sólo aquí, sino también en la small talk de los investigadores se equipara con frecuencia a la racionalidad por excelencia. De hecho, numerosas publicaciones se ciñen a la "racionalidad" como tema de la teoría de la acción, sin mencionar siquiera sus aspectos cognitivos. La fascinación de lo nuevo y lo moderno que emana de la teoría de la racionalidad ha impedido a la mayoría de los teóricos de la racionalidad buscar en el acervo de la filosoffa tradicional de la razón motivos que puedan resultar útiles en contextos actuales. De este modo permaneció fuera de consideración gran parte de la rica experiencia reflexiva que el pensamiento del pasado acumuló en este campo. Una consecuencia de ello es la tendencia de casi todos los teóricos de la racionalidad a darse por satisfechos con una muy rudimentaria precomprensión de la racionalidad como capacidad de fundamentación, como si el animal rationale no pudiese hacer muchas otras cosas aparte de fundamentar, por ejemplo pensar, actuar, reflexionar, meditar, resolver problemas, jugar, etc.

((0.3)) No hay ninguna razón para ocuparse únicamente, en una teoría de la racionalidad, de lo que por razones históricas y contingentes queda de la razón, como el que fuera el tema central de la filosofía, en las teorías contemporáneas de la racionalidad. Creo que una teoría de este tipo debería proponerse como meta reunir, ante todo, las diversas líneas de la discusión, y esto sólo será posible si se las analiza tratando de encontrar los conceptos y motivos fundamentales comunes a todas ellas. Desde esta perspectiva será posible revelar los estrechamientos a que se ve sometida la cuestión de la racionalidad en cada una de las líneas de discusión examinadas, y evitarlas en la construcción de la propia teoría. A continuación es indispensable lograr una orientación general en el ámbito del acervo de la tradición, pero también en el campo de nuestras intuiciones (por ejemplo, la diferencia entre lo racional (rational) y lo razonable (vernünftig) en los usos lingüísticos actuales). En tercer lugar esta teoría de la racionalidad debería proponrse la ambiciosa meta de ser realmente comprehensiva y tematizar, en la medida de lo posible, todo lo que constituye la rationalitas del animal rationale.

$((0.4))$ Por "teoría de la racionaliad" entiendo una teoría analítica, es decir, el intento de llevar a cabo un análisis sistemático del concepto de racionalidad sobre el trasfondo de los contextos ejemplares de su aplicación. Pero 
una teoría de este tipo sólo es posible con medios tipológicos, y su núcleo no contendrá mucho más que una tipología de la racionalidad. Las tipologías resultan inevitables allí donde el campo de los fenómenos es demasiado complejo para llevar a cabo una construcción teórica en sentido estricto, mediante axiomas o definiciones, y éste es realmente el caso por lo que respecta al tema "racionalidad». Ni en la tradición ni en los discursos contemporáneos sobre la racionalidad existe un explicandum único, y a ello se añade que los propios conceptos que han de servir de guía tienen también su historia: la virtud dianoética de la «epistémen de Aristóteles no puede traducirse simplemente por "conocimiento científico" en el sentido que nosotros damos a esta expresión, y a la inversa, en la filosofía práctica de este filósofo no hay nada que corresponda al tipo ideal de la racionalidad económica de Mill, porque en ella «oikonomía significa algo diferente. Pero el argumento más importante contra el carácter teóricamente riguroso de la teoría filosófica de la racionalidad procede de su imperfección de principio: una teoría de la racionalidad debe ser ella misma racional, es decir que en el curso de su análisis de las formas de racionalidad no puede analizar la forma de racionalidad que ella misma sigue precisamente en ese momento. Siempre queda detrás o se sustrae algo inanalizable o no objetivable. Esta imperfección puede tener inconvenientes, pero garantiza el carácter abierto en general del concepto de racionalidad y la capacidad de aprendizaje de la propia teoría de la racionalidad, que nunca podrá presentarse como un edificio teórico acabado. Este carácter abierto debe ser fundamentado con más detalle en el contexto mismo de la teoría de la racionalidad.

\section{Fundamentaciones}

((1.1)) Sigo, en primer lugar, la opinión tradicional, y también hoy muy extendida, de que la racionalidad se caracteriza esencialmente por la capacidad de fundamentar, donde la expresión "fundamentación" no ha de entenderse, por lo pronto, mucho más que la respuesta a las preguntas que comienzan con la interrogación "¿por qué?». Es sorprendente que las modernas teorías de la racionalidad que siguen este hilo conductor refieran la fundamentación casi sin excepción a opiniones, convicciones, propósitos o decisiones y dejen de lado lo que la tradición, por lo menos hasta Kant, entendió unánimemente por 
"fundamentar»: la aducción de fundamentos (o razones) (Grïnde) ${ }^{1}$ y causas de los estados y acontecimientos del mundo. Si se quiere incluir este aspecto de la racionalidad en la teoría, hay que comenzar con una tipología de las fundamentaciones en el nivel del lenguaje objeto ${ }^{2}$ (objektsprachliche Begründungen). En este punto es apropiado partir de los pares distintivos "objetivo/subjetivo" así como "teórico/práctico", de los que resultan cuatro tipos de fundamentación en el nivel del lenguaje objeto:

(1) Fundamentaciones objetivo-teóricas o cognitivas, como respuestas a la pregunta: “ppor qué (es) p (el caso)?” ('p' por el contenido proposicional de un enunciado).

(2) Fundamentaciones objetivo-prácticas o normativas, como respuestas a la pregunta: «por qué ha de hacerse p?».

(3) Fundamentaciones subjetivo-teóricas o epistémicas, como respuestas a la pregunta: "¿por qué se acepta, se supone, se espera, etc. (por ejemplo, tú), que p?"

(4) Fundamentaciones subjetivo-prácticas o intencionales, como respuestas a la pregunta: “jpor qué tú (por ejemplo) haces/hiciste p?»

\section{((1.2)) Observaciones.}

Ad (1): Las fundamentaciones cognitivas constituyen lo que de Aristóteles a Hegel se entendió por «ciencia»: el conocimiento de lo universal, de los fundamentos y las causas, entendidos estos términos en el nivel del lenguaje objeto, es decir que el científico conoce la esencia de las cosas y con ello sus fundamentos y causas ${ }^{3}$. La "ciencia" en este sentido siempre fue, desde la Antigüedad, el paradigma de la racionalidad en general.

1 El término alemán Grund equivale en español tanto a "fundamento" como a "razón". Este segundo sentido alude a la motivación racional, mientras que el primero puede entenderse casi como un sinónimo de causa, y ser aplicado a sucesos del mundo. No sin ambiguiedad, el autor maneja indistintamente el término Grund en ambos sentidos. Cuando su uso me parece ambiguo, introduzco las dos traducciones posibles $(N$. del $t$.).

2 Por supuesto, estas fundamentaciones se tratan en la teoría moderna de la ciencia bajo el rótulo de "explicaciones». Desde el punto de vista de la historia de esta terminología, la expresión "explicación" es tan joven como "fundamentación".

3 El rationem reddere como traducción de lógon te doûnai kaì déchesthai ha de entenderse primariamente en el nivel del lenguje objeto, así como el modelo aristotélico de la apódeixis. . 
Ad (2) y (4): Entiendo las acciones como la realización de p, en la que considero importante el carácter proposicional de "p". La omisión puede concebirse como una subclase de esta realización.

Ad (2): Las fundamentaciones normativas se refieren a los fundamentos (o razones) objetivos por los que algo debe hacerse. No distingo entre normas de validez incondicional (categórica) y condicional (hipotética); en el primer caso se trata de normas morales en sentido estricto, mientras que las restantes normas pueden clasificarse como prescripciones técnicas (en el sentido de la téchne) y reglas de la prudencia (Klugheitsregeln).

Ad (3): Las fundamentaciones epistémicas ${ }^{4}$ son subjetivas en el sentido de que operan desde el punto de vista del sujeto en relación con un p que, sin embargo, mienta un estado de cosas objetivo. Si alguen afirma que en Japón se ha producido un terremoto, se remitirá a las variaciones del sismógrafo para fundamentar su afirmación, sin afirmar con ello que estas variaciones sean la causa del terremoto. Igualmente procederá al hacer un pronóstico meteorológico con ayuda de un barómetro. Las fundamentaciones epistémicas presentan, pues, la causa/ratio cognoscendi de $\mathrm{p}$ y no la causa/ratio essendi de $\mathrm{p}$, como las fundamentaciones cognitivas. (La distinción terminológica unívoca entre causa y ratio se encuentra por primera vez en Leibniz, y desde entonces se ha vertido al alemán mediante la diferencia entre causas y fundamentos (o razones)). La convicción o la expectativa de que p se fundamenta por referencia a un nexo objetivo-causal entre cambios de estado en el mundo y signos identificables de esos cambios; pero no es, ella misma, el explanandum de una fundamentación causal o explicación, porque las variaciones del sismógrafo o del barómetro, que por su parte son causadas (kausal verursacht) por $\mathrm{p}$, no causan (kausal verursachen) la convicción o la expectativa de que $\mathrm{p}$, sino que sólo las sugieren a título de "buenas razones" (condiciones del antecedente) de un argumento de fundamentación epistémica.

Ad (4): Las fundamentaciones intencionales no se refieren a los fundamentos (o razones) objetivos para hacer algo, fundados por ejemplo en el contexto de un sistema de normas válido o en la "naturaleza de la cosa», sino a los fundamentos (o razones), motivos, propósitos, etc., que tiene alguien que hace algo o tiene el propósito de hacerlo. Es paradigmático de este tipo de funda-

4 La expresión procede de Carl G. HEMPEL. 
mentaciones el «silogismo práctico». Desde el punto de vista del observador, las fundamentaciones intencionales constituyen la base del concepto weberiano de explicación comprensiva en las ciencias sociales. Weber caracteriza la sociología como "una ciencia que comprende la acción social mediante una interpretación y pretende explicarla causalmente en su desarrollo y en sus efectos" ${ }^{5}$. En la discusión en torno a este género de fundamentación, discusión desarrollada bajo el rótulo de "explicación racional de la acción" (rationale Handlungserklärung), los defensores de la unidad de la ciencia se obstinaban en que en realidad sólo se trata aquí de una variante del modelo de explicación causal de Hempel-Oppenheim, lo cual no es correcto. Primero ha de comprenderse la intención de la acción en el contexto del "sentido subjetivo" (Max Weber) del agente, antes de que se pueda plantear la cuestión de si esa intención también es o fue fácticamente la causa de la acción en cuestión.

((1.3)) De este modo resultan cuatro tipos de racionalidad de fundamentación en el nivel del lenguaje-objeto: la racionalidad cognitiva, la racionalidad normativa, la racionalidad epistémica y la racionalidad intencional (también "práctica» en sentido restringido).

\section{((1.4)) Observaciones:}

(a) En Aristóteles encontramos que la racionalidad cognitiva y la normativa se presentan como las virtudes dianoéticas epistéme, téchne y asimismo phrónesis, que quedan comprometidas con el punto de vista objetivo porque Aristóteles las caracteriza como un modo del aletheúein, es decir, del encuentro de lo verdadero. Para la racionalidad epistémica y la intencional no se encuentran en este filósofo propuestas terminológicas, sino a lo sumo la alusión al logismós (la reflexión) que diferencia al hombre del animal, tal como se dice en la Política.

(b) Para Aristóteles, como para Kant, la diferencia entre razón tórica y razón práctica es esencial, si bien no hay correspondencia en la obra de Aristóteles con la distinción kantiana entre lo técnico-práctico y lo moral-práctico, a no ser que se entienda la conciencia de un deber categórico como una conciencia noética, es decir, en el sentido del noûs como facultad intelectual intuitiva.

5 Max WEBER, Gesammelte Aufsätze zur Wissenschaftslehre, 4. Auflage, Tübingen 1974, pg. 542. 
(c) Así como Kant intenta reconstruir en general la objetividad de lo cognitivo y lo normativo partiendo de condiciones subjetivas de posibilidad, puede entenderse su crítica de la razón como el intento de presentar la racionalidad cognitiva como un caso especial comprobable de la racionalidad epistémica, y la racionalidad normativa como un caso especial comprobable de la racionalidad intencional. También en esto sigue Kant el paradigma filosófico cartesiano que comienza con la duda y por ello sólo dispone para la ciencia, en un principio, de fundamentos desde el punto de vista subjetivo. En general, la racionalidad de fundamentación corresponde a lo que Kant entiende por "razón" en sentido restringido, es decir, diferenciándola de la facultad de juzgar y del entendimiento: la facultad de establecer conclusiones, de la conexión de juicios y luego también de los principios.

\section{Argumentación}

((2.1)) Con este término me refiero a las fundamentaciones en el marco de aquellos contextos discursivos en los cuales las razones que se aducen no se refieren inmediatamente a los estados de cosas en tanto que estados de cosas existentes, o que deban producirse, o presumirse, o convertirse en propósitos, etc., sino a las fundamentaciones mismas en el nivel del lenguaje objeto que se refieren a dichos estados de cosas, y más concretamente a las pretensiones de validez vinculadas a dichas fundamentaciones. En este punto adopto la terminología de Jürgen Habermas: «llamamos argumentación al tipo de discurso en el que los participantes tematizan ciertas pretensiones de validez que se han vuelto objeto de disputa e intentan saldarlas y criticarlas con argumentos. Un argumento contiene razones que están conectadas de forma sistemática con la pretensión de validez de un enunciado problematizado" ${ }^{6}$. Es característica de la argumentación el entrelazamiento de crítica y justificación ${ }^{7}$. Si se acepta la distinción entre acción comunicativa (primaria) y discurso, se pueden reunir las formas argumentativas de fundamentación en el tipo de la "fundamentación discursiva" y caracterizar en general la racionalidad de la argumentación como racionalidad discursiva.

6 Jürgen HABERMAS, Theorie des komunikativen Handelns I, Frankfurt/Main 1981, 38

7 o.c., 38 y ss. 


\section{((2.2)) Observación:}

No siempre es fácil determinar la diferencia entre las fundamentacions en el nivel del lenguaje objeto y las fundamentaciones en el nivel del metalenguaje (o entre explicaciones y argumentaciones). Naturalmente, ' $p$ porque $q$ ' $o$ ' $h$ está prohibido porque i está prohibido' son también argumentos, pero por regla general no decimos que alguien "argumenta" que el sol ha calentado la piedra, o que se debe pagar los impuestos a causa de las leyes vigentes. A menudo se llama "argumentación" a la aducción de razones epistémicas o intencionales, aunque en estos casos se trata también de explicaciones: ¡es realmente la variación del sismógrafo un argumento de que en Japón se ha producido un terremoto? ¿O es la intención de hacer algo determinado un argumento para hacerlo? En tal caso, según la acepción amplia del concepto de argumentación sería argumentativo todo discurso en el que algo se muestra, o se demuestra, o se explica, o se hace plausible, etc. (lat. arguo: exponer claramente, mostrar, demostrar). La argumentación y la fundamentación serían sencillamente lo mismo, y la teoría de la argumentación abarcaría toda la lógica así como la dialéctica aristotélica (tópica), es decir, el arte de la fundamentación en el nivel del lenguaje objeto sobre la base de premisas imperfectas. Por ello, si se quiere dar al término "argumentación" un contorno más preciso, es aconsejable reservarlo para el metanivel, al menos topológicamente. En tal caso, en la acción discursiva "argumetación" no se trata primariamente de estados de cosas que se afirman como existentes, se presumen, se esperan, etc., o bien deben o no deben realizarse, sino que se trata del hecho de afirmar, presumir, esperar ciertos estados de cosas etc., o bien del hecho de realizarlos o no realizarlos, y más concretamente de las pretensiones de validez vinculadas a estos actos o entabladas por ellos. Y también las expresiones "crítica» y ujustificación" tienen aquí un sentido más preciso, pues la filosofía, desde Kant (por lo menos), llama "críticas" precisamente a las argumentaciones de este tipo ${ }^{8}$.

((2.3)) Tales pretensiones de validez están vinculadas, en primer término, a las fundamentaciones en el nivel del lenguaje objeto, y así en la argumenta-

8 Nadie critica que el sol sale cada mañana, pero se puede criticar la afirmación de que el sol sale cada mañana, es decir que en el ámbito teórico la "crítica" está localizada en el metanivel. En el campo práctico, en cambio, el discurso versa también sobre la crítica o la justificación de hechos o situaciones políticas o sociales, pero con ello se alude a posicionamientos o juicios de valor normativos que, en tanto que acciones discursivas, mantienen la misma relación con la crítica argumentativa que todas las restantes acciones discursivas. 
ción se trata en primer témino de si tales fundamentaciones son sólidas, convincentes o astringentes. Por ello, a los cuatro tipos de racionalidad en el nivel del lenguaje objeto se atribuyen sendos proyectos de lógicas que indagan las condiciones formales de la astringencia de cada una de las formas de fundamentación: las lógicas clásica, deóntica, epistémica e intencional. Estas lógicas no se ocupan de $\mathrm{p}$, $\mathrm{q}$ o r, sino de ' $\mathrm{p}$ ', ' $\mathrm{q}$ ' o ' $\mathrm{r}$ ' atendiendo a la astringencia de sus conexiones en las condiciones de determinados caracteres de validez que se presuponen (por ejemplo, 'verdadero/falso', 'ordenado/prohibido/permitido', etc.), pero respecto de los cuales dichas lógicas mismas no se posicionan.

La argumentación lógica sólo puede poner en juego las condiciones formales de astringencia de las fundamentaciones. En cambio, su astringencia material se mide por el carácter de validez de las afirmaciones parciales, efectuadas en el nivel del lenguje objeto y contenidas en dichas fundamentaciones. Así, una fundamentación cognitiva determinada (explicación causal) de p sólo será correcta y concluyente si al menos se da $\mathrm{p}$, es decir, si ' $\mathrm{p}$ ' es verdadero. Lo mismo vale decir de las afirmaciones acerca de las condiciones del antecedente y de las afirmaciones de leyes con cuantificador universal. Las fundamentaciones normativas, por ejemplo 'p ha de realizarse' se miden por la pretendida corrección de la realización de $\mathrm{p}$ en un determinado contexto normativo, donde aún habría que distinguir entre lo bueno en sentido evaluativo y el mandato prescriptivo o el carácter permitido de las acciones. En el ámbito epistémico se trata de la plausibilidad del enunciado 'p' en cuestión, y el ámbito intencional se caracteriza por la pretensión de sentido (Sinvollsein) de una realización de $\mathrm{p}$ (en su correspondiente contexto de discurso epistémico e intencional).

\section{((2.4)) Observaciones:}

(a) En esta reconstrucción nos referimos en primer lugar, y trivialmente, sólo a las pretensiones de validez que se saldan discursivamente. Pero también llevamos a cabo discursos acerca de pretensiones de validez que no pueden saldarse completamente por medios discursivos (por ejemplo, en el ámbito evaluativo subjetivo o en el ámbito estético) que no por ello son simplemente irracionales. El ámbito de la racionalidad es, de todos modos, más amplio que el de las fundamentaciones en el nivel del lenguaje objeto y las fundamentaciones discursivas. Sus límites se precisarán más adelante.

(b) En la fundamentación cognitiva y epistémica se trata de p, en la fundamentación discursiva, en cambio, de 'p', respecto de su verdad y plausibili- 
dad. La disputa entre científicos es por lo general argumentativa en este sentido, es decir que no se cuestionan los hechos y sus nexos objetivos, sino las afirmaciones que se refieren a ellos. Así, resulta convincente caracterizar con Popper la ciencia moderna en su conjunto como una empresa crítica, de la que, claro está, no se puede excluir la justificación, compañera necesaria de la crítica, por el hecho de que pueda asociarse a ella el dogmatismo o la "fundamentación últiman: donde no se pretende validez alguna y, por ello, nada se justifica argumentativamente, tampoco hay nada que criticar.

(c) Existe una asimetría entre las fundamentaciones teóricas y las fundamentaciones prácticas, pues sólo puede ser tematizado el contenido proposicional de las fundamentaciones teóricas y las afirmaciones parciales contenidas en ellas en relación con las pretensiones de validez "verdad" o "plausibilidad", mientras que su función ilocucionaria sigue, como toda acción, la pretensión de validez «rectitud» o «sentido». Para el discurso práctico, esto significa lo siguiente: en las fundamentaciones normativas e intencionles se dice por qué ha de realizarse p o por qué alguien realiza, ha realizado o tiene la intención de realizar p. En el nivel discursivo se trata de clarificar si es correcto o si tiene sentido realizar p, es decir, el objeto de discurso que se pone en cuestión no es, pues, el propio $\mathrm{p}$ que ha de realizarse, sino la realización de $\mathrm{p}$, atendiendo a la rectitud o el sentido, y esto es exactamente el nivel comunicativo de la ética, a diferencia de la moral. La moral dice: "debe hacerse p" y pretende corrección para la relización de $\mathrm{p}$, las mayoría de las veces recurriendo a otras afirmaciones normativas en el nivel del lenguaje objeto. En la ética se trata de clarificar si esta pretensión es lícita. La ética pregunta: "¿es realmente correcto hacer p?", y sólo puede aclarar esta cuestión si es capaz de aducir buenas razones para la propia afirmación «debe hacerse p" y para las premisas de las que se sigue. Esta es la diferencia ntre una fundamentación moral y una justificación ética.

(d) En las fundamentaciones intencionales, con la pretensión de validez "sentido" (Sinnvollsein) no nos referimos a una comprensibilidad hermenéutica, sino pragmática, en el sentido de un sentido reproducible de la acción (nachvollziehbarer Handlungssinn) (Max Weber). Sin tal atribución/suposición de sentido no podríamos identificar como acción un suceso. En este punto es importante distinguir con precisión entre las perspectivas subjetiva y objetiva de sentido. El último Max Weber señaló con exactitud esta diferencia distinguiendo la «racionalidad objetiva de corrección» (objektive Richtigkeitsrationalität) y la "racionalidad subjetiva de fines" (subjektive Zwecksrationalität). Conforme a esta distinción, podemos atribuir más o 
menos sentido a la realización de $\mathrm{p}$ aunque no podamos o queramos tomar partido por lo que respecta a su correción objetiva.

((2.5)) La tematización de las pretensiones de validez "verdadero", "correcto", "plausible» y "con sentido», que pueden saldarse discursivamente con afirmaciones y acciones, define el campo de la racionalidad discursiva. Esta se caracteriza esencialmente por la argumentación, entendida como alternancia de crítica y justificación. Kant hace de la racionalidad discursiva entendida en este sentido, como "crítica", la clave de la racionalidad científica y moral en general, y Popper le sigue en este punto con su racionalismo crítico del "trial and erron" o de las "conjectures and refutations", que extiende, más allá de los límites de la teoría de la ciencia, a la filosofía social. La racionalidad crítica en este sentido es una racionalidad del enjuiciamiento según criterios, y Kant era todavía consciente de la procedencia forense de este modelo cuando comparó la crítica de la razón con un tribunal. Ahora bien, es importante el hecho de que este enjuiciamiento según criterios sigue otras reglas que las del juicio y la fundamentación de juicios en el nivel del lenguaje objeto. Kant describió con mucha precisión esta lógica del enjuiciamiento y con ello llamó la atención sobre ciertas consecuencias muy importantes para cualquier teoría de la racionalidad.

En el caso de las fundamentaciones cognitivas en el nivel del lenguaje objeto, por ejemplo $p$ se funda en que $q$, y en que siempre $p$ cuando $q$. No puedo fundar que ' $p$ ' es verdadero afirmando que $q$, sino sólo que ' $q$ ' es verdadero, $y$ que ' $p$ ' es siempre verdadero cuando ' $q$ ' es verdadero, es decir que la fundamentación discursiva permanece en un principio en el metanivel. Pero, ¿̨cómo puedo establecer que el propio 'p', o 'q', o 'r', ... es verdadero? Sin duda, no será averiguando si "p' es verdadero" es a su vez verdadero, pues esto conduce a un regreso infinito, sino sólo mediante un criterio de verdad, por ejemplo "p' es verdadero si y sólo si p" (Tarski), criterio que refiero a un 'p', ' $q$ ', 'r', ... en el enjuiciamiento crítico. Esta capacidad de aplicar un universal a un particular o de pensar un particular como contenido en un universal («subsumir») fue caracterizada por Kant como lo específico de la facultad de juzgar como facultad racional. Por eso afirmo que la facultad de juzgar, así caracterizada, es el órgano y el medio de la argumentación en el sentido de la alternancia de crítica y justificación, o de la racionalidad discursiva en general.

Desde una perspectiva histórica puede mostrarse que Kant, en la Crítica de la facultad de juzgar, no sólo reflexionó sobre las condiciones de posibilidad de 
los juicios estéticos y teleológicos, sino también sobre los fundamentos del quehacer de la crítica de la razón en general. La tercera crítica no se limita a vincular posteriormente la razón teórica y la razón práctica, sino que tematiza lo que la crítica es en esencia: enjuiciamiento de pretensiones de la razón a la luz de criterios universales. Para ello cuenta Kant con la facultad de juzgar determinante, así como con la reflexionante. Si en el primer caso los puntos de vista universales de la «subsunción» están dados previamente, en el segundo han de averiguarse reflexivamente. No se puede pasar por alto que las propias críticas de Kant siguen sin excepción el modo reflexionante de la facultad de juzgar, y cuando pretenden ser determinantes, porque creen poder recurrir a la lógica como ciencia cerrada, la física newtoniana y el "factum de la razón pura práctica" como lo universal dado previamente, esta pretensión se funda en argumentos igualmente reflexionantes que la historia posterior de la filosofía y de la ciencia han refutado.

((2.6)) La conexión de racionalidad discursiva y facultad de juzgar tiene importantes consecuencias. Hay que señalar, con Kant, que «la lógica general de la facultad de juzgar no puede dar prescripciones» ${ }^{9}$, pues hay que definirla como "la facultad de subsumir bajo reglas, es decir, de discernir si algo se algo se encuentra o no bajo una regla dada (casus datae legis)» ${ }^{10}$. No puede haber reglas para esto, porque su aplicación requeriría a su vez una regla, y así hasta el infinito ${ }^{11}$. Pero si la racionalidad discursiva es esencialmente un asunto de la facultad de juzgar, en tanto que facultad racional, esto significa que el contexto de la crítica y la justificación nunca puede ser acotado completamente por reglas, aun cuando debe seguir, indudablemente, ciertas reglas elementales (por ejemplo, las de la gramaticalidad o la consistencia lógica). Por consiguiente, una lógica de la argumentación nunca será posible como teoría puramente axiomático-deductiva o simplemente como teoría cerrada a priori. Por lo que respecta a la argumentación misma, de ello se sigue que toda argumentación, examinada con detalle, contendrá siempre ciertos elementos de estimación y decisión. Los enjuiciamientos no son deducibles como las demostraciones lógicas o matemáticas, sin ser por ello simplemente "irracionales". Sólo ha de exigirse que en el contexto de crítica y justificación las decisión

$9 \mathrm{KrV}, \mathrm{B} 174$

$10 \mathrm{KrV}, \mathrm{B}$ 179. La expresión latina certifica el contexto forense de introducción del concepto kantiano de facultad de juzgar.

11 Cf. B 171 y ss. 
misma de estimar algo de este modo y no de otro permanezca por principio expuesta al enjuiciamiento crítico. Con ello se satisfacen los imperativos de la racionalidad discursiva.

\section{((2.7)) Observaciones:}

(a) Esta apertura de principio propia de la racionalidad discursiva no resulta, empero, sólo de la imposibilidad de establecer reglas fijas de aplicación de reglas, sino también de la inabarcabilidad a priori de las reglas mismas, cuando entra en juego la facultad de juzgar reflexionante: las reglas de la argumentación aceptadas por nosotros nos nos prescriben cómo hemos de aplicarlas al caso particular, ni nos prescriben cuáles otras reglas de argumentación habremos de aceptar cuando ya no basten las que hemos aceptado hasta ahora. También aquí entran en juego la estimación y la decisión, y tal vez nos veamos obligados a encontrar o inventar otras reglas mediante el modo reflexionante de la facultad de juzgar.

(b) Con esto queda señalado el lugar en que entran en juego las otras pretensiones de validez, muy numerosas, que entablamos en el discurso y la acción, y que hasta ahora no han sido objeto de consideración. Nos referimos ante todo a las pretensiones de validez no saldables discursivamente, acerca de las cuales, no obstante, intentamos llegar discursivamente a un entendimiento. La racionalidad discursiva es más amplia qu el ámbito de lo que puede saldarse discursivamente.

(c) Éste es también el lugar de la rcionalidad en tanto que pretensión de validez, es decir, del predicado "racional» en tanto que predicado normativo que empleamos cuando juzgamos algo como racional o irracional. Es evidente que se pretende que estos juicios mismos son racionales, con lo cual pueden convertirse a su vez en el tema de un discurso, con el que sucede a su vez lo mismo. En tal caso, en este nivel discutimos conceptos criteriológicos alternativos de racionalidad ${ }^{12}$.

(d) Este carácter irreductiblemente abierto de la racionalidad discursiva es, por último, la razón de la incompletud sistemática de toda teoría de la racionalidad que deba operar asimismo como teoría analítica en el nivel del discurso: una teoría de este tipo no puede en modo alguno proceder de otro

12 Por ejemplo, el de razón «transversal» (Welsch). 
modo que reconstruyendo en el elemento de la facultad de juzgar reflexionante las reglas paradigmáticas de aplicación del predicado "racional" y juzgando críticamente sus aplicaciones fácticas a la luz de estas o aquellas reglas justificadas como racionales, con lo que los modos fácticos de aplicación alternativa de las reglas pueden motivar también la crítica de determinadas construcciones de reglas.

(e) En esta situación hemos ganado una argumentación acerca de nuestras reglas de argumentación, es decir que la racionalidad discursiva se ha revelado no sólo como racionalidad abierta, sino también como completamente reflexiva ${ }^{13}$. Es exactamente este hecho lo que Kant tiene en mente en el famoso doble sentido del título "Crítica de la razón pura»: la razón criticada y la razón que critica son una y la misma; la razón sólo puede extraer de sí misma los criterios de la crítica de la razón, es decir que ha de obtenerlos en el elemento de la facultad de juzgar reflexionante, de donde resulta (contra la autocomprensión de Kant), el carácter irreductiblemente abierto del discurso crítico de la filosofía. Cualesquiera patrones y criterios de racionalidad discursiva que los filósofos puedan descubrir, aunque sean los más universales y fundamentales, están expuestos a una disposición crítica, siempre que los filósofos conduzcan su discurso como un discurso crítico. En este sentido, criticismo y falibilismo son realmente inseparables.

(f) De lo anterior se sigue un argumento contra el proyecto de una fundamentación última reflexiva en el elemento de la reflexión trascendental, proyecto que pasa por alto que precisamente la reflexividad completa de la racionalidad discursiva no puede significar su clausura (Geschlossenbeit) en algún "núcleo" trascendental. El mero hecho de que este proyecto se discuta argumentativamente muestra que un set de reglas de argumentación cerrado de una vez y para siempre, que habría aceptado "ya siempre" todo el que argumenta, simplemente no existe. El que, empero, cree en ello, no puede hacer otra cosa que reprochar a sus oponentes escépticos en la argumentación su ceguera o su malevolencia (o ambas cosas). En realidad, en toda argumenta-

13 Como ha mostrado sobre todo Apel, la reflexividad completa es incompatible con una superposición infinita de meta-niveles y meta-meta-niveles. No obstante, la distinción antre lenguaje objeto y metalenguaje sigue siendo útil con fines analíticos, siempre que no se olvide que sólo tiene sentido en un lenguaje natural que siempre sigue siendo su propio y más elevado metalenguaje. 
ción no sólo pueden ser siempre objeto de debate las aplicaciones de las reglas de argumentación aceptadas, sino también, y por principio, estas reglas mismas, y han sido y son transformadas con medios argumentativos. La teoría kantiana de la facultad de juzgar y su aplicación a la teoría de la argumentación nos enseñan que no se puede vincular el grado de astringencia lógica de las inferencias deductivas a los argumentos estrictamente reflexivos de la fundamentación última, ya sólo porque se trata de argumentos en el sentido de la racionalidad discursiva, es decir, del contexto de crítica y justificación, que precisamente no está limitado completamente por reglas.

\section{Reglas}

((3.1)) Si la racionalidad discursiva formula juicios referidos a las pretensiones de validez vinculadas a las fundamentaciones en el nivel del lenguaje objeto y a las afirmaciones parciales contenidas en dichas fundamentaciones, entonces el ámbito de la racionalidad misma no puede comenzar allí donde se entablan de hecho las pretensiones de validez, sino que debe abarcar también aquello a lo que pueden vincularse en general las pretensiones de validez. La capacidad de formular estos aspirantes a validez (en el terreno, todavía neutral, de su conexión en forma de fundamentaciones) debe ser ya una facultad racional, pues en caso contrario el hiato entre lo racional y lo irracional sería insalvable. (Lo mismo cabe decir de las fundamentaciones en el nivel del lenguaje objeto, en relación con las afimaciones parciales contenidas en ellas). Para Kant, los juicios y las acciones son candidatos de este tipo, y su denominador común es la "facultad de los conceptos", siendo la facultad teórica de los conceptos el entendimiento que produce juicios, y siendo la «facultad de desear según conceptos» la voluntad, en tanto que causa de las acciones. Si se acepta la caracterización kantiana, más precisa, de la facultad de los conceptos como "facultad de las reglas" (y tiene todas las razones para hacerlo quien sea nominalista e interprete los conceptos como reglas del uso de las expresiones predicativas), se puede concebir, en una primera aproximación, las facultades elementales racionales del habla ${ }^{14}$ y la acción, a las que aluden los términos "entendimiento" y "voluntad", como facultades de seguir reglas.

14 Asumo en este punto, con Platón y Kant, y sin otra fundamentación, que el pensamiento es lenguaje internalizado. 
El seguimiento de reglas es algo más que la mera regularidad del comportamiento; ésta por sí sola no sería condición suficiente para conectar el seguimiento de reglas con la racionalidad. El comportamiento regular (es decir, instintivamente guiado por reglas) de los animales es indistinguible de la mera legaliformidad (Gesetzesartigkeit), y en este ámbito tampoco hay nada que entender, sino sólo que explicar. Lo que distingue el seguimiento de reglas del comportamiento meramente regular es la capacidad de disponer de las reglas, es decir, que el seguimiento de reglas es reflexivo. Sólo es capaz de seguir reglas el ser vivo que puede distinguir los casos en los que sigue una regla de aquellos otros en los que la contraviene, lo cual presupone una conciencia de esta regla. Pero si una regla es consciente, entonces también es posible contravenirla, es decir, que por principio el comportamiento se desvíe de ella. La falibilidad y la libertad (en el sentido de disponer de alternativas) se revelan, pues, como condiciones elementales de la racionalidad en general, si se interpreta la racionalidad a partir del concepto de regla. Pero la capacidad de disponer de reglas no se agota en la posibilidad de contravenir consciente e intencionadamente una regla, sino que alude también a la facultad de hallar e inventar reglas que a su vez pueden referirse (en tanto que meta-reglas) a la formación y aplicación de reglas del comportamiento. La posibilidad formal de proseguir indefinidamente la superposición de meta-meta-reglas no significa otra cosa que la imposibilidad de principio de reducir completamente a reglas la aplicación de reglas (Kant), es decir que la racionalidad elemental de reglas, por principio reflexiva, ya es ella misma un concepto abierto, y no sólo la facultad de juzgar o la racionalidad discursiva.

((3.2)) Pero el seguimiento de reglas es una condición elemental de la comprensibilidad del comportamiento. La razón de ello es el hecho de que son imposibles las reglas puramente privadas, por lo que el comportamiento guiado por reglas apunta ya siempre a una intersubjetividad posible. Por eso quien sigue reglas ha ingresado ya en el campo de lo comprensible, pues al hacerlo ejemplifica algo que ya siempre comparte potencialmente con otros. Pero este compartir con otros no atañe sólo a la posibilidad de ser comprendido por otros, sino también a la de comprender a otros, con lo cual el carácter potencialmente común de las reglas tiende también aquí un puente: lo que las teorías románticas de la comprensión describían como "introafección" o "empatía» alude en realidad a la facultad de interpretar el comportamiento ajeno a la luz de una regla que también podría ser propia. Comprender significa, pues, la capacidad y actividad de asumir reglas hipotéticamente frente a algo que hay 
que comprender. Dado que el seguimiento de reglas implica también la posibilidad de disponer de las reglas en el sentido señalado más arriba, esta capacidad de disposición misma debe satisfacer las condiciones de comprensibilidad, es decir que debemos concebir de antemano la racionalidad de reglas en su totalidad como una facultad, por principio intersubjetiva, de lo comprensible y de la comprensión. A ella pueden vincularse en la reconstrucción tanto la racionalidad de las fundamentaciones en el nivel del lenguaje objeto como la racionalidad discursiva de la argumentación.

((3.3)) La racionalidad de reglas constituye, pues, el ámbito central y básico de la racionalidad en general, pues proporciona aquello a lo que remiten las fundamentaciones y las argumentaciones. La fundamentación en el nivel del lenguaje objeto responde a preguntas introducidas por la interrogación “ipor qué?» que se refieren por su parte a un 'p' en el modo de la afirmación, la prescripción, la suposición, etc., o en el modo de tener la intención de hacerlo. La base y el punto de partida son aquí actos de habla simples cuya comprensibilidad se constituye y limita por la racionalidad de reglas. Dado que seguir una regla implica siempre la capacidad de disponer de la regla, entra en juego la posibilidad de principio de desacertar (Misslingen) a causa de la infracción de una regla, infracción que, por su parte, en el marco de la racionalidad de reglas, sólo puede ser identificada y "subsanada" mediante meta-reglas. Tampoco aquí pueden excluirse nuevas infracciones, etc., de modo que la diferencia «aciertodesacierto" recorre todo el ámbito de la racionalidad de reglas. Pero esta diferencia abre el campo de aquello a lo que se refiere secundariamente la racionalidad discursiva: el ámbito de las pretensiones de validez en el sentido de diversas diferencias de validez. La articulación de un 'p', en tanto que contenido proposicional de actos de habla, según reglas sintácticas y semánticas de referencia y predicación puede acertar o desacertar en la misma medida que el comportamiento lingüístico conforme a una determinada función ilocucionaria, y esto vale también, a fortiori, para acciones lingüísticas tan complejas como son todo tipo de fundamentaciones en el nivel del lenguaje objeto. La pretensión de todo acto de habla, es decir, lo que en él se supone como dado, es su acierto (incluso allí donde, irónica o lúdicamente, la intención es el desacierto del acto de habla, el desacierto debe acertar), y dado que este acierto se determina por reglas, siempre está vinculada a él la pretensión de validez de la corrección: el acto de habla debe tenerse por acertado conforme a ciertas reglas, es decir, como "conforme a las reglas" o correcto. El par "correcto-incorrecto" (richtig-falsch) corresponde aquí al par "acertado-desacertado». 
((3.4)) El par acierto-desacierto traza, pues, el diseño de la diferencia de validez de aquello que pretenden los actos de habla en general, pero con la corrección conforme a reglas no parecen satisfacerse otras dos pretensiones de validez que igualmente se vinculan a los actos de habla: el éxito y la verdad. La pretensión de tener éxito se refiere al efecto perlocucionario al que apunta el acto de habla, y como en todas las otras acciones orientadas al éxito, hablamos de acierto cuando se produce el efecto que se pretendía. Con respecto al efecto perlocucionario, los actos de habla coinciden con todas las acciones orientadas al éxito (Habermas) que no son acciones lingüísticas. La pregunta, entonces, es si el éxito es una pretensión de validez vinculada a las acciones orientadas al éxito. Pero es evidente que tales acciones pueden resultar desacertadas por razones que no ha de justificar el hablante o agente. Ciertas condiciones de partida desconocidas pueden impedir el éxito de la acción en la misma medida que ciertos nexos causales (Wirkungszusammenhänge) desconocidos que se entrometan en el curso de la misma. La falta de éxito, pues, no califica sin más de incorrecta (falsch) o irracional a una acción orientada al éxito, sino que lo hace solamente si se relaciona con el saber causal disponible en cada caso. Cuando en una partida de ajedrez quiero dar jaque a mi contrincante, debo hacerlo "correctamente", es decir, conforme a reglas, pero cuando talo un cerezo, no tengo éxito según ciertas reglas, sino a la luz del saber causal: si hiciese algo incorrecto, arremetería contra él armado con una lima de uñas. No obstante, si se quiere relacionar esta incorrección o irracionalidad con la pretensión de validez de la corrección, hay que distinguir los tipos de reglas que son pertinentes en cada caso: en el ajedrez se trata del éxito conforme a reglas constitutivas, y en la tala del árbol se trata del éxito a la luz de reglas regulativas (técnicas) ${ }^{15}$. Llamaré «acción performativa» a la acción que sigue reglas constitutivas.

Con la modificación señalada se puede asignar a la pretensión de validez de la corrección no sólo la acción performativa (hablar, en sentido ilocucionario, jugar al ajedrez, tocar música con partitura, bailar un minué, bucear, practicar un exorcismo, etc.), sino también la acción orientada al éxito, y no hay duda de que en el nivel de la racionalidad discursiva (es decir, de la argumentación)

15 En la distinción de los tipos de reglas sigo a Searle. La «racionalidad objetiva de corrección" de Max Weber admite ambas interpretaciones: puede aludir a la corección en el sentido de la conformidad con reglas de acción válidas o que se suponen válidas, o a la corrección en el sentido de la elección adecuada de medios a la luz del saber causal disponible. 
se tematizan en general ambos géneros de esta corrección pretendida. Ha de retenerse que no sólo los actos de habla que intervienen en fundamentaciones y argumentaciones son candidatos a juicios de corrección (hasta aquí ha podido parecer que ésta era el caso, por razones relacionadas con la estructura de la reconstrucción que aquí se ha escogido), sino todas las acciones, por cuanto que se emprenden guiadas por reglas. Con tales acciones se entabla igualmente, independientemente de que se hable de ello, una pretensión de corrección que, por supuesto, también es verbalizable paralelamente a las emisiones explicitamente performativas, como es la expresión "ijaque!" que acompaña a una jugada de ajedrez conforme a las reglas.

Pero, ¿qué sucede con la pretensión de verdad? La pretensión de verdad se entabla con el contenido proposicional de los actos de habla, es decir que la afirmación ' $p$ !' no puede ser verdadera ni falsa, sino que sólo puede serlo el 'p' afirmado ${ }^{16}$. La diferencia de validez "verdadero-falso" entra en juego también en las presuposiciones lógicas y pragmáticas de los actos de habla (el imperativo "iobedece al emperador alemán!" carece de sentido si la proposición "hay un emperador alemán» es falsa), pero normalmente sólo interviene en el acto de habla de la afirmación. Pero en teoría de la verdad se discute si el carácter verdadero de la afirmación puede concebirse como un género de la corrección conforme a reglas. Para dirimir esta cuestión haría falta un criterio de verdad indiscutido, pero no existe un criterio tal. Los objetivistas o realistas acentúan la irreductibilidad de la verdad a la corrección de los actos de habla, mientras que los pragmatistas y los defensores de teorías discursivas aproximan ambas pretensiones de validez equiparando la verdad a lo que en determinadas situaciones puede afirmarse como correcto, es decir, conforme a reglas aceptadas.

((3.5)) No podemos dirimir aquí la disputa de si la verdad se agota en la "afirmabilidad racional" (Putnam) o en algún otro tipo de corrección conforme a reglas. En nuestro contexto es importante solamente la circunstancia de que el ámbito de la racionalidad de reglas es más amplio que el de la verdad: quien dice algo falso o miente no es irracional ya sólo por eso, pues sólo los seres racionales pueden en general decir algo verdadero o falso (errar o mentir). Lo mismo puede decirse de las restantes pretensiones de validez, es decir que la racionalidad en el sentido elemental de racionalidad de reglas no es sólo la capacidad de decir algo que sea falso o verdadero, sino la de hacer algo en

16 Cf. las teorías de la verdad de STRAwSON y Habermas. 
general que en sentido potencialmente reflexivo sigue ciertas reglas y puede ser enjuiciado como acertado o desacertado a la luz de las reglas correspondientes.

La racionalidad de lo dicho y lo hecho ha sido caracterizada más arriba como comprensibilidad; ahora es aconsejable añadir la diferencia entre la comprensión del sentido y la comprensión de la acción. Sin la comprensión de un 'p' como representación posible de un estado de cosas y de un suceso del mundo (por ejemplo, el movimiento de un cuerpo) en tanto que acción, no hay nada en absoluto a lo que puedan referirse las afirmaciones, suposiciones, etc., por un lado, y las prescripciones e intenciones, por otro lado. Hay que reparar en que la condición de constituido por reglas de este algo comprensible extiende sobre él una "retícula" al menos de tres modos: las reglas del decir constituyen un todo porque sólo se puede decir algo en una lengua, y las reglas del hacer forman parte ya siempre de un saber total institucional y causal, en el que los elementos del saber se interpretan mutuamente. En segundo lugar, no se puede mantener completamente separadas la racionalidad del decir y la del hacer, porque el decir o hablar es también hacer y, a la inversa, la capacidad de acción remite a situaciones intencionales o proposicionales tales como deseos, intenciones, etc. que, por su parte, no pueden ser identificadas independientemente del medio simbólico de su representación ${ }^{17}$. El tercer holismo afecta a la capacidad discursiva del ser racional, que se sigue, por su parte, de la reflexividad de principio del seguimiento de reglas. Estas condiciones de la reflexión, que no pueden cerrarse, inscribirse en un modelo concluso, son también el fundamento del carácter abierto de la racionalidad, pero también de que sea imposible abarcarla en su totalidad con la mirada, lo cual obliga a la teoría a establecer tipologías.

\section{Intencionalidad}

((4.1)) La racionalidad de reglas es, pues, una totalidad abierta que afecta a los aspectos de decir, dar a entender (meinen) ${ }^{18}$ y hacer conectados entre sí en la regla, y que constituye el fundamento de aquello que hace del hombre un ani-

17 Cf. el apartado 4.

18 El verbo alemán meinen aparece alternativamente en sus significados de «dar a entender" y de "mentar" (N. del t.). 
mal rationale. El seguimiento de reglas potencialmente reflexivo es al mismo tiempo la base de la comprensibilidad intersubjetiva de lo que los hombres dicen, dan a entender y hacen, y en este sentido la racionalidad de reglas inaugura también la comunicabilidad de lo humano. Si se quiere, puede llamarse a esto "racionalidad comunicativa", pero hay que tener en cuenta que el ámbito de lo comprensible es mucho más amplio que el de la acción comunicativa, es decir, orientada al entendimiento (Habermas): también es comprensible el seguimiento de reglas potencialmente reflexivo que no se orienta por el télos del entendimiento o del acuerdo. A ello se añade la circunstancia de que es posible comprender y seguir reglas independientemente de toda verbalización (por ejemplo, en el comportamiento mimético o en la comunicación no verbal), de tal modo que lo comunicativo de la racionalidad de reglas puede ser independiente no sólo de la orientación teleológica hacia el entendimiento, sino también del lenguaje como canal. Desde este punto de vista, la orientación hacia el entendimiento o hacia el éxito y la racionalidad de fundamentación, tanto discursiva como en el nivel del lenguaje objeto, pueden interpretarse como especificaciones de la racionalidad de reglas en determinadas condiciones restrictivas.

Así, pues, la racionalidad es ante todo la facultad de lo comprensible, la capacidad de hacer, de dar a entender y de entender algo comprensible. Cuando se intenta comprender este tipo elemental de racionalidad desde sus propias condiciones elementales, es natural preguntar cómo ha de caracterizarse con mayor precisión la facultad de decir, dar a entender o hacer algo, con lo que la atención se dirige hacia el fenómeno de la intencionalidad. Es evidente que la intencionalidad, como la facultad humana de referirse a algo en el modo de mentar (meinen), creer, suponer, temer, tener la intención (siendo la intención de la acción nada más que un caso especial de la intencionalidad), etc., no es lo mismo que la racionalidad, pero es, ciertamente, un aspecto insoslayable de esta competencia. Interpretar a un ser vivo como animal rationale exige por lo menos poder atribuirle situaciones intencionales, pero ello no es aún condición suficiente para la atribución de racionalidad, si la entendemos como racionalidad de reglas. La disputa en torno a las teorías intencionales del significado (Grice, etc.) mostró que las reglas o las convenciones no están contenidas analíticamente en las intenciones de significación, lo que significa, a la inversa, que es posible dirigirse intencionalmente a algo que no sigue ninguna regla. Los niños juegan a menudo de esa forma, y muestran con ello que también el concepto de acción es más amplio que el de comportamiento que sigue reglas, a no ser que se niegue que los niños que juegan actú- 
an de este modo. Ahora bien, no atañe a nuestro asunto si las reglas/convenciones pueden (Lewis) o no pueden derivarse de intenciones. Sólo es importante la relación no analítica entre intenciones y reglas, que constituye la diferencia entre intencionalidad y racionalidad.

Los intencionalistas afirman que se puede comprender perfectamente lo que alguien dice o hace si se comprende lo que mienta. Según esto, la representación lingüística conforme a las convenciones comunicativas sería secundaria. Los convencionalistas, en cambio, afirman que no se puede mentar absolutamente nada independientemente del medio comunicativo que es el lenguaje: “¿Cómo puedo saber lo que miento antes de escuchar lo que digo?». Realmente los intencionalistas se ven en la dificultad de tener que explicar cómo alguien puede mentar p (por ejemplo, que llueve ${ }^{19}$ ) sin disponer de la proposición "llueve» y emplearla en el acto de mención. En cambio los convencionalistas, que pretenden vincular la totalidad de las situacones intencionales a la capacidad de disponer de las reglas de un lenguaje natural, se ven obligados a rehusar toda situación intencional a los niños antes de la adquisición del lenguaje, a quienes padecen minusvalías relacionadas con el lenguaje, a los afásicos, a los primates y a todos los animales superiores. El recuerdo de Peirce ofrece aquí una salida, pero también el de Cassirer y el de Susanne Langer, que nos dieron la posibilidad de disociar la intencionalidad de su vínculo demasiado estricto con los lenguajes verbales y de darnos por satisfechos con algo más elemental en la explicación de la posibilidad de los actos de referirse a algo o de tener la intención de hacer algo. Hay que dar la razón a los convencionalistas cuando insisten en que sólo se puede mentar o tener la intención de hacer algo como algo. Además, lo mentado y aquello que nos proponemos debemos siempre mentarlo o proponérnoslo como algo que mentamos o nos proponemos en un modo determinado ${ }^{20}$, pero no es necesario (tal es mi tesis) que este medio (Medium) del mentar-algo-como-algo-en-un-determinado-modo deba ser un lenguaje natural; basta cualquier medio simbólico. $\mathrm{Si}$ a esto se añade la distinción de Susanne Langer ${ }^{21}$ entre símbolos discursi-

19 Asumo aquí, sin fundamentarlo, que lo intencional es siempre proposicional. Esto no se discute tampoco en el debate entre intencionalistas y convencionalistas.

20 Cf. sobre esto la reinterpretación mentalista de los roles ilocucionarios en J. R. SEARLE, Intentionalität (ed. alemana) Frankfurt/Main 1983.

21 Cf. Susanne K. LaNGer, Philosophie auf neuem Wege, ed. alemana de Philosophy in a New Key (1942), Frankfurt/Main 1984, especialmente págs. 84 yss. 
vos y presentativos, se abre una pluralidad de universos simbólicos en los que los hombres pueden dar a entender, referirse a o tener la intención de algo con algo, de múltiples modos. Los pintores, compositores, mimos y muchas otras personas que trabajan en medios no verbales documentan con hechos la amplitud del campo intencional, que ciertamente es muy amplio, pero, precisamente, no se llena completamente con la intencionalidad lingüística. $\mathrm{Ni}$ siquiera está dada la posibilidad de principio de verbalización, pues ¿qué se puede verbalizar en la novena sinfonía de Beethoven? La pluralidad de mundos simbólicos es otra razón de la pluralidad inabarcable e inacotable a priori de los fenómenos y tipos de racionalidad.

((4.2)) Si realmente la intencionalidad y el empleo de símbolos van emparejados, tenemos buenas razones para pensar, con Cassirer, el animal rationale como animal symbolicum. Ambas determinaciones serían equivalentes si se superpusiesen completamente los ámbitos de la racionalidad de reglas y del empleo de símbolos, pero sobre esto hay dudas. Sobre todo Susanne Langer llamó la atención sobre el hecho de que los símbolos presentativos no siguen, por regla general, una sintaxis estricta, dependen en buena medida del contexto y, por tanto, no son tampoco traducibles. No obstante, obras plásticas abstractas como, por ejemplo, las de Arp o Moore, que no transportan ninguna información disociable de la contemplación, son símbolos en el sentido de Cassirer y Langer: articulaciones sensibles cargadas de «sentido". Dado que, como es obvio, los mundos simbólicos ostentan una mínima articulación sintáctica y semántica de sus elementos, puede hablarse de racionalidad simbólica, siguiendo la delimitación que hemos establecido aquí. Cabe esperar la réplica de si no puede en absoluto pensarse una función simbólica que carezca por su parte de los elementos de tal articulación sintáctico-samántica, pero esta réplica habría de dirigirse a una teoría detallada de los símbolos.

La función simbólica fundamental es la de representación simbólica, y puede mostrarse que es fundamental para los elementos de la racionalidad de reglas. $\mathrm{Si}$ es correcto que no se puede mentar algo simplemente (ya sea en el sentido de la representación, ya en el de la intención), sino sólo algo como algo $\mathrm{y}$, además, en un determinado modo, entonces la propia estructura de "como algo" característica del mentar remite a lo simbólico de lo representado en el mentar: si miento algo como algo, nunca puede aquello como lo cual miento algo estar meramente en lo mentado, sino que debe ser representado simbólicamente. Pero el algo-como-algo no es otra cosa que la estructura proposicional del propio p. La representación simbólica es tanto la base del contenido 
proposicional de los actos de habla (es decir, de la unidad de referencia y predicación) como de la posibilidad de proponerse efectos ilocucionarios y perlocucionarios mediante un uso específico de predicaciones. La acción con sentido, es decir, la acción que se supone comprensible, es igualmente impensable si no se asume un "sentido subjetivo" (Max Weber), que por su parte debe ser representable simbólicamente para poder ser vinculado al comportamiento intencionado o manifiesto.

\section{La conexión con la antropología}

((5.1)) Ya la figura clásica del animal rationale conectaba la filosofía de la razón con la antropología, en el sentido de doctrina filosófica del hombre. Platón y Aristóteles sitúan su teoría de las virtudes dianoéticas en el contexto del conocimiento del alma humana, aun cuando posteriormente proyecten las características de ésta, en modo eminente, a los dioses o a lo divino. Kant se niega, ciertamente, a fundamentar la filosofía de la razón en la antropología y, así, habla también de «seres racionales en general». La razón de ello es el hecho de que la crítica de la razón no puede ser sustituida por una teoría objetiva de lo racional, pues en la primera se trata del cercioramiento reflexivo de la razón que llevamos ya a toda teoría objetiva y hacemos valer en ella. Sin embargo, Kant no deja lugar a dudas de que esta razón trascendental de principios es la nuestra: finita, es decir, en el plano del conocimiento, encerrada dentro de los límites de la sensibilidad posible, discursiva y no intuitiva; y, en la acción, eficaz sólo de forma limitada. Al idealismo alemán hay que reprocharle haber desdeñado estas condiciones restrictivas de una filosofía trascendental de la razón. Su pretensión hipertrófica de poder filosofar desde la perspectiva de la razón absoluta es aún hoy la caja de resonancia de la crítica totalizadora de la razón de nuestros días, pues Hegel y Nietzsche pertenecen a un mismo género. Por ello el reestablecimiento del vínculo entre la teoría de la racionalidad y la antropología filosófica no es mero sentimentalismo hacia la historia de la filosofía, sino que sirve a un doble objetivo: evitar que esta teoría recaiga en un idealismo de tipos puros de racionalidad, es decir, tipos que en modo alguno puedan encontrar ejemplo en nuestro pensamiento y nuestra acción; y, por otro lado, reaccionar contra las consecuencias derrotistas de la crítica totalizadora de la razón mostrando claramente que somos seres que realmente pueden ser a la vez racionales y humanos. La rehabilitación del animal rationale debe- 
ría asegurar la humanidad (Menschlichkeit) de la razón y al mismo tiempo reaccionar contra la tendencia de moda de buscar lo humano allí donde no está la razón.

((5.2)) En la obra tardía de Cassirer encontramos ya establecida la conexión de la teoría de la racionalidad con la antropología filosófica: en la antropología del animal symbolicum. Para desarrollar este tránsito con mayor precisión hace falta, a mi juicio, dar dos pasos metódicos a los que aquí simplemente aludiremos. En primer lugar, las intuiciones de Cassirer en teoría de los símbolos deben ser elaboradas con más precisión, lo que sólo es posible mediante la combinación de su teoría de la "pregnancia simbólica" con la semiótica de Charles S. Peirce. Pero de la categoría de "terceridad» se produce el tránsito, nada forzado, a la "posicionalidad excéntrica" de Helmuth Plessner como determinación antropológica fundamental. Sus elementos o "leyes" ("artificialidad natural», «inmediatez mediata», y "localización utópica») permiten comprender cómo un ser que las ejemplifica no puede representar e interpretar el mundo abierto en el que vive de otro modo que como mundo simbólico: en el modo del algo-como-algo, es decir, a la luz de un ser nunca meramente natural, en un estar siempre ya más allá de lo inmediato, en la «mirada de ninguna parte» (Nagel). Dado que la teoría de la racionalidad debe asentar sus fundamentos sobre una teoría de la representación simbólica que remite por su propio sentido a la antropología del animal symbolicum, sólo ésta es la antropología de la razón adecuada a nuestro tiempo. 